\title{
Metabolic acidosis in late pregnancy due to 5-oxoproline (pyroglutamic acid)—A case report
}

\author{
Sahathevan Sathiyathasan", K. Jeyanthan, R. Hamid \\ Mayday University Hospital Croydon, Surrey, UK \\ Email: " Sathya@doctors.org.uk, Kannappar.Jeyanthan@mayday.nhs.uk, Rosol.hamid@mayday.nhs.uk
}

Received 21 November 2011; revised 29 December 2011; accepted 16 January 2012

\section{ABSTRACT}

Introduction: Accumulation of 5-oxoproline (pyroglutamic acid) is a rare cause of severe, high anion gap metabolic acidosis in adults. Case: A 21 year old lady presented at 39 weeks gestation in her first pregnancy with 2 weeks history of shortness of breath. She suffered from ear ache and had been taking Paracetamol on regular basis for a year. She was admitted to having regular alcohol intake until the pregnancy when she stopped. She was not in acute distress and all her observations were stable. The laboratory analysis of renal function was normal. Arterial blood gas showed metabolic acidosis. The anion gap $34 \mathrm{mEq} / \mathrm{l}$ which was consistent with metabolic acidosis. Plasma aminoacid screen revealed no abnormality. Chest $\mathrm{X}$-rays showed patchy consolidation keeping with a chest infection. Emergency caesarean section was carried out for fetal distress in first stage of labour. Urine and serum samples were taken which showed an increase in the serum level five Oxoproline to creatinine ratio indicating pyroglutamic metabolic acidosis. Polyglutamate was found in the urine She was admitted to intensive care unit; cardiovascular stable, had no signs of sepsis. She underwent ventilation and haemofiltration. On second day of admission metabolic acidosis was corrected. During the 48 hours treatment with supportive therapy produced general overall improvement. Discussion: Metabolic acidosis that caused by 5-oxoproline results from disruption of the gamma glutamyl cycle. Glutamile synthetase (GS) deficiency is an autosomol recessive disorder. With GS deficiency, reduced glutathione levels increase gamma glutamile synthetase activity, and the resulting gamma-glutamile cystine levels are particularly converted to 5-oxoproline. Intoxication of organic acids is a differential diagnosis of high anion gap metabolic acidosis with no renal impairment, there was no evidence of ethanol, methanol or ethylene glycol ingestion. Suspicion for 5-

${ }^{*}$ Corresponding author. oxoproline-associated high anion gap metabolic acidosis should be entertained when the cause of high anion gap metabolic acidosis remains poorly defined, the anion gap cannot be explained reasonably by measured organic acids, and there is concomitant acetaminophen use. Conclusion: Clinicians need to be aware of this unusual cause of anion gap acidosis because it may be more common than expected, early discontinuation of the offending agent is therapeutic, and administration of $\mathrm{N}$-acetylcysteine could be beneficial.

Keywords: Metabolic Acidosis; Anion Gap; Acetaminophen; Pyroglutamic Aciduria; Pregnancy

\section{INTRODUCTION}

A rare cause of high anion gap acidosis is 5-oxoproline (pyroglutamic acid), an organic acid intermediate of the $\gamma$-glutamyl cycle. Polyglutamic academia should be considered when most common causes such as ketoacidosis, lactic acidosis or ingestion of salicylates, methanol or ethynyl glycol were excluded [1].

\section{CASE}

A 21 years old lady presented at 39 weeks gestation in her first pregnancy with 2 weeks history of shortness of breath. She suffered from earache and had been taking Paracetamol on regular basis for a year. She was admitted twice during the course of her pregnancy. She was initially treated for UTI with Amoxicillin and subsequently followed by treatment of chest infection with Erythromycin. She had regular alcohol intake until she found out she was pregnant. There was no past medical history of diabetes, hypertension or renal disease.

She was not in acute distress with a normal respiratory rate and blood pressure except her pulse rate was 105 $\mathrm{bpm}$. The baseline laboratory analysis revealed her haemoglobin was $10.3 \mathrm{~g} / \mathrm{dl}$ and high MCV. Arterial blood gas showed metabolic acidosis (Table 1). Her white cell 
count was 20,500/ul. Blood urea, creatinine and electrolytes were all within normal limits at that time. Liver enzymes showed increased gamma glutamyl transferase level of $202 \mathrm{u} / \mathrm{l}$. The anion gap $34 \mathrm{mEq} / 1$ was consistent with metabolic acidosis (normal range should be less than $13 \mathrm{mEq} / \mathrm{l}$ ). Plasma amino acid screen revealed no abnormality. Plasma x-glutamyl transferase level was $202 \mathrm{u} / 1(6-42)$.

Chest X-rays showed patchy consolidation in keeping with chest infection. On admission, she was put on continuous fetal heart tocographic (CTG) monitoring. At that stage, the CTG showed unprovoked decelerations and emergency caesarean section was carried out.

Since she had no renal impairment, the differential diagnosis for high anion gap metabolic acidosis was intoxication due to organic acids but there was no evidence of ethanol, methanol or ethylene glycol ingestion.

Urine and serum samples were taken which showed an increase in the serum level five Oxoproline to Creatinine ratio indicating pyroglutamic metabolic acidosis. Polyglutamate was found in the urine.

She was then admitted to intensive care unit. Haemodynamically she was stable and she had no signs of sepsis. She was ventilated and had haemofiltration. Her metabolic acidosis was corrected on the following day. She recovered uneventfully within the 48 hours of supportive therapy.

\section{DISCUSSION}

Pyroglutamic metabolic acidosis is a condition which can be caused by pregnancy, anorexia, alcohol abuse, chronic Paracetamol ingestion, liver or renal disease. Polyglutamic academia (5-oxoprolinemia) occurs as a result of overproduction of polyglutamic acid secondary to deficiency of glutathione synthetase (GS) which causes the failure of breakdown of polyglutamic acid by 5-oxoprolinase [1].

Several inherited enzyme defects that have been charecterised are extremely rare and usually presents at a very early age with neurologic and heamatologic abnormalities. GS deficiency is an autosomol recessive disorder. With

Table 1. Arterial blood gas result.

\begin{tabular}{cc}
\hline \multicolumn{3}{c}{ Blood Gas } \\
\hline $\mathrm{pH}$ & 7.26 \\
$\mathrm{pCO}_{2}$ & $1.05 \mathrm{kPa}$ \\
$\mathrm{pO}_{2}$ & $26.0 \mathrm{kPa}$ \\
$\mathrm{HCO}^{3-}$ & $8.9 \mathrm{kPa}$ \\
Base Excess & -23.6 \\
Lactate & $1.6 \mathrm{kPa}$ \\
\hline
\end{tabular}

GS deficiency, reduced glutathione levels increase $\mathrm{x}$ glutamile synthetase activity and the resulting $\mathrm{x}$-glutamile cystine levels are particularly converted to 5oxoproline (Figure 1).

We believe our patient may have had heterozygote for glutathione synthetase deficiency; heterozygote state is charecterised by a moderate reduction $(50 \%)$ in the activity of glutathione synthetase with normal baseline glutathione levels and no clinical abnormality [2]. Acquired 5-Oxoprolinuria has been described in adults several contributory factors include malnutrition, pregnancy and strict vegetarian diet [3]. Severe 5-oxoproline aciduria and academia have been described in patients who use several different medications these include acetaminophen (Paracetamol), Vigabatrin and the antibiotics Flucloxacillin and Netilmicin [4].

High anion gap metabolic acidosis occurs frequently in patients with acetaminophen toxicity. This generally is attributed to lactic acidosis and kidney failure. However in some cases anion gap cannot be explained. These patients often have less severe toxicity and history of chronic acetaminophen ingestion [5].

In a literature review of 18 adult cases of 5-oxoprolinuria and high anion gap metabolic acidosis are associated with chronic therapeutic ingestion of acetaminophen. Interestingly, 14 out of 18 described patients were women. The activity of several isoenzymes in the $\mathrm{x}$ glutamyl cycle is known to be different in men and women. These differences may increase the susceptibility to women to develop this disorder [6]. Serious hepatotoxicity at recommended or near recommended doses for therapeutic purposes has been reported in association with chronic alcohol use as chronic alcoholics are predispose to Paracetamol related toxicity at relatively low doses [7]. This again was possible contributing factor in this case.

Enhanced toxicity of acetaminophen abusers of alcohol and the drugs can be expected for several reasons: First, in human studies, acute alcohol intake has been shown to decrease toxic metabolic activation (mixed function oxidase system) of Paracetamol in liver micrososmes [8]. Second, poor diet in the alcoholic may enhance the toxicity of acetaminophen. Third, pre-existent liver disease may increase the risk of toxic liver injury. Finally, the alcoholics may be more likely than the general public to exceed the recommended doses of overthe-counter drugs [9].

Sepsis or sepsis like systemic inflammatory response can be associated with pyroglutamic acidemia; this is explained by animal studies. Liver glutathione can be depressed in established septic shock. This will reduce feedback inhibition of x-glutamyl cysteine synthetase, allowing the increased production of pyroglutamic acid 


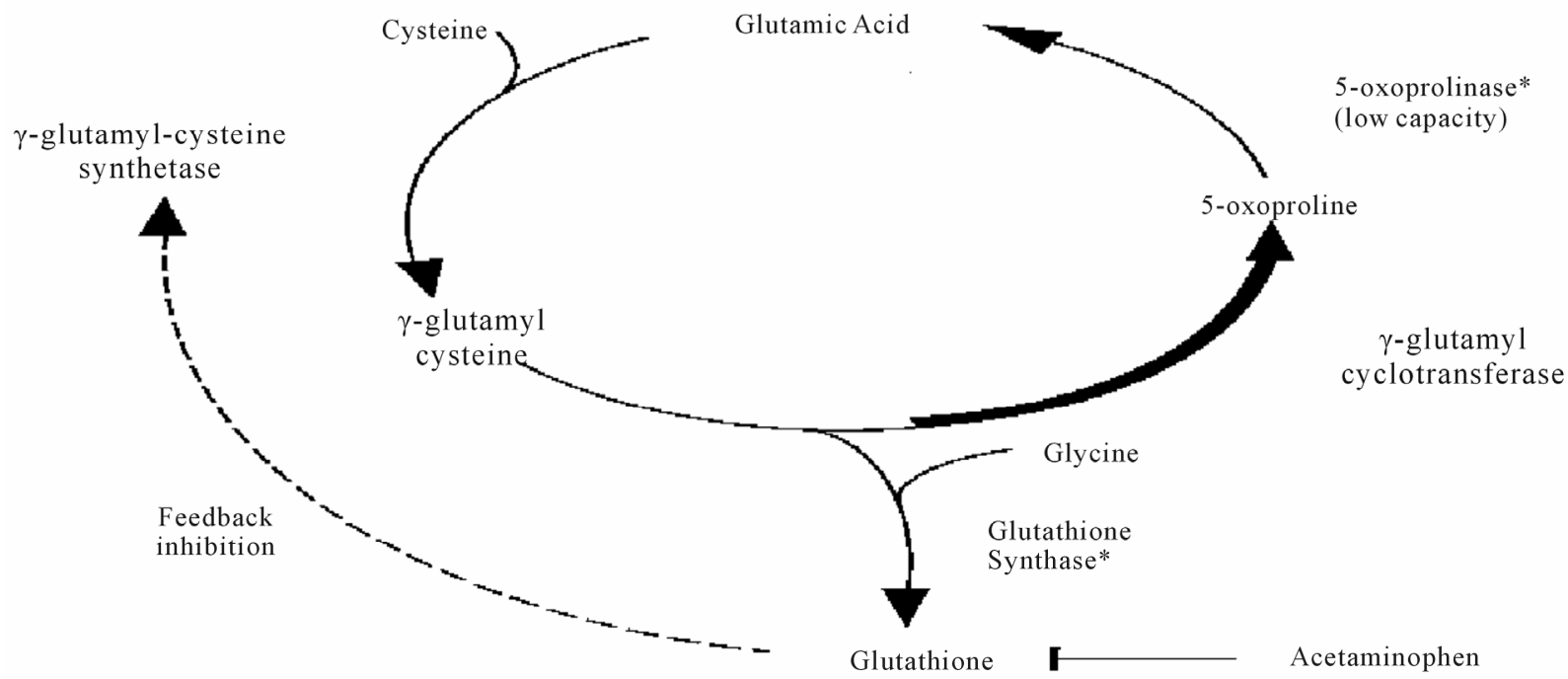

Figure 1. The x-glutamyl cycle: 5-oxoprolineisanorganicacidintermediate, acetaminophen reduces intracellular glutathione levels (1).

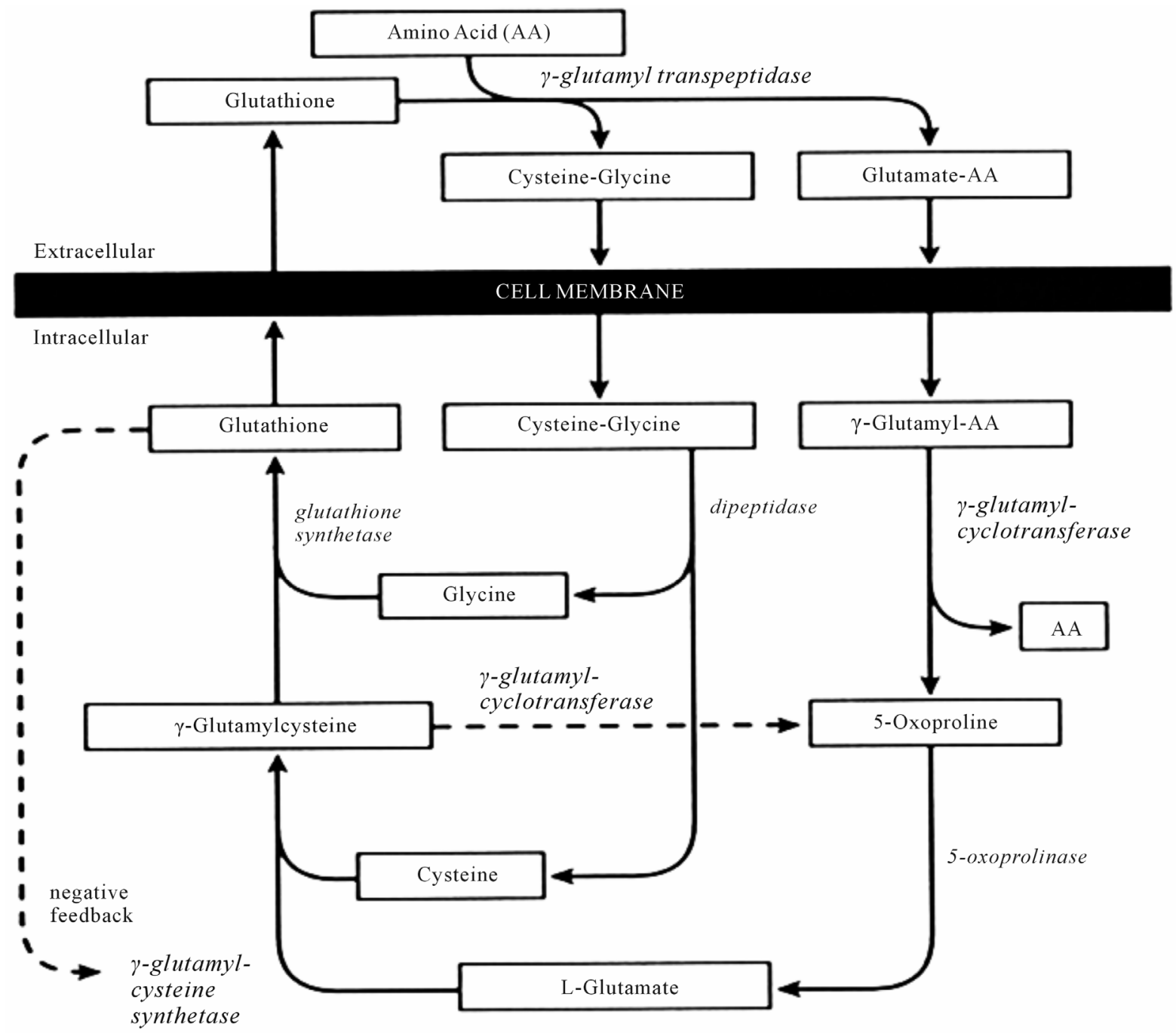

Figure 2. Gamma-glutamyl cycle (2). 
and cysteine [10] (Figure 2).

Polyglutamic academia may be seen in association with pregnancy when glycine availability is limited; generally, the levels of polyglutamate are much less marked in these conditions than those described in the inherited disorders of metabolism [1].

Metabolic acidosis could lead to fetal acidosis and electrolyte imbalance [11]. There were studies also showed increasing metabolic acidosis was accompanied by decreasing rate of blood flow in the uterine circulation [12]. These causes could have contributed to fetal distress in this case.

Management of severe polyglutamic acidemia involves primarily in supportive measures with the aim of preservation and optimization of respiratory, cardiovascular and renal functions. The presence of infection should be sought and treated accordingly.

\section{CONCLUSIONS}

Acetaminophen, a popular non-prescription analgesic and antipyretic drug, is available in various dosage levels as generic compound as well as in combination with other agents. The chronic use of over the counter medication could potentially be hazardous in certain group of populations. For example, long term use of acetaminophen even at therapeutic doses can lead to complications in chronic alcoholics. Moreover these patients could have been heterozygote for glutathione synthetase deficiency which increases the risk for toxicity. Obtaining detailed drug history and personal history are significantly important to assess the risk even with acetaminophen which is a commonly used drug in the absence of other metabolic causes.

Clinicians need to be aware of this unusual cause of anion gap acidosis because it may be more common than expected. Early discontinuation of the offending agent is therapeutic and administration of $\mathrm{N}$-acetylcysteine could be beneficial.

\section{REFERENCES}

[1] Gerard, A., Dempsey, F.R.C.A., Heather, J. and Lyall, B. (2000) Polyglutamic academia: A cause of high anion gap metabolic acidosis. Critical Care Medicine, 28, 1803-1807. doi:10.1097/00003246-200006000-00018
[2] Spielberg, S.P. (1984) In vitro assessment of pahrmacogenic susceptibility to toxicto toxicv drug metabolites in humans. Federation Proceedings, 43, 2308-2313.

[3] Fenves, A.Z., Kirkpatrick III, H.M. and Patel, V.K.V. (2006) Increased anion gap metabolic acidosis as a result of 5oxopronoline (Pyroglutamic acid): A role for Acetaminophen. Clinical Journal of American Society of Nephrology, 1, 441-447. doi:10.2215/CJN.01411005

[4] Pitt, J. (1990) Association between Paracetamol and pyroglutamic aciduria. Clinical Chemistry, 36, 173-174.

[5] Record, C.O., IIles, R.A., Cohen, R.D. and Williams, R. (1975) Acid base and metabolic disturbances in fulminant hepatic failure. Gut, 16, 144-149. doi:10.1136/gut.16.2.144

[6] Butera, L., Feinfeld, D.A. and Bhargava, M. (1990) Sex differences in the subunits of glutathione-S-transferase isoenzyme from rat and human kidney. Enzyme, 43, 176182.

[7] Riordan, S.M. and Williams, R. (2002) Alcohol exposure and Paracetamol-induced hepatotoxicity. Addiction Biology, 7, 191-206. doi:10.1080/13556210220120424

[8] Tummel, K.E., Slattery, J.T. and Nelson, S.D. (1989) Effect on ethanol on hepatotoxicity of acetaminophen in mice and reactive metabolic formation by mouse and human liver micrososmes. Toxicology and Applied Pharmacolpgy, 100, 391-397. doi:10.1016/0041-008X(89)90287-1

[9] Michael, H., Leist, M.D., Lawrence, E., Gluskin, M.D., John, A. and Payne, M.D. (1985) Enhanced toxicity of acetaminophen in alcoholics: Report of three cases. Journal of Clinical Gastroenterology, 7, 55-59. doi:10.1097/00004836-198502000-00008

[10] Colomb, V., Petit, J., Matheix-Fortunet, H., et al. (1995) Influence of antibiotics and food intake on liver glutathione and cytochrome P-450 in septic rats. British Journal of Nutrition, 73, 99-110. doi:10.1079/BJN19950012

[11] Kamalakannan, D., Baskar, V. and Barton, D.M. (2003) Diabetic ketoacidosis in pregnancy. Postgraduate Medical Journal, 79, 454-457. doi:10.1136/pmj.79.934.454

[12] Blechner, J.N., Stenger, V.G. and Prystowsky, H. (1975) Blood flow to human uterus during maternal metabolic acidosis. American Journal of Obstetrics \& Gynecology, 121, 789-794. 